\title{
Curcumin regulates cell fate and metabolism by inhibiting hedgehog signaling in hepatic stellate cells
}

\author{
Naqi Lian', Yuanyuan Jiang ${ }^{1}$, Feng Zhang ${ }^{1,2,3}$, Huanhuan Jin' ${ }^{1}$ Chunfeng Lu', Xiafei Wu', Yin Lu ${ }^{1,2,3}$ and \\ Shizhong Zheng ${ }^{1,2,3}$
}

Accumulating evidence indicates that Hedgehog $(\mathrm{Hh})$ signaling becomes activated in chronic liver injury and plays a role in the pathogenesis of hepatic fibrosis. Hepatic stellate cells (HSCs) are Hh-responsive cells and activation of the Hh pathway promotes transdifferentiation of HSCs into myofibroblasts. Targeting Hh signaling may be a novel therapeutic strategy for treatment of liver fibrosis. We previously reported that curcumin has potent antifibrotic effects in vivo and in vitro, but the underlying mechanisms are not fully elucidated. This study shows that curcumin downregulated Patched and Smoothened, two key elements in Hh signaling, but restored Hhip expression in rat liver with carbon tetrachlorideinduced fibrosis and in cultured HSCs. Curcumin also halted the nuclear translocation, DNA binding, and transcription activity of Gli1. Moreover, the Hh signaling inhibitor cyclopamine, like curcumin, arrested the cell cycle, induced mitochondrial apoptosis, reduced fibrotic gene expression, restored lipid accumulation, and inhibited invasion and migration in HSCs. However, curcumin's effects on cell fate and fibrogenic properties of HSCs were abolished by the Hh pathway agonist SAG. Furthermore, curcumin and cyclopamine decreased intracellular levels of adenosine triphosphate and lactate, and inhibited the expression and/or function of several key molecules controlling glycolysis. However, SAG abrogated the curcumin effects on these parameters of glycolysis. Animal data also showed that curcumin downregulated glycolysis-regulatory proteins in rat fibrotic liver. These aggregated data therefore indicate that curcumin modulated cell fate and metabolism by disrupting the Hh pathway in $\mathrm{HSCs}$, providing novel molecular insights into curcumin reduction of HSC activation.

Laboratory Investigation (2015) 95, 790-803; doi:10.1038/labinvest.2015.59; published online 4 May 2015

Liver fibrosis associated with significant morbidity and mortality worldwide represents a wound repair process secondary to liver pathological responses to all forms of chronic injuries. Chronic liver injury transforms quiescent hepatic stellate cells (HSCs) that are activated and develop a myofibroblast-like phenotype acquiring many fibrogenic properties including overgrowth, loss of lipid droplets, and enhanced motility during fibrogenesis. ${ }^{1}$ Recent evidence reveals that HSCs undergo rapid energy reprogramming during activation and their survival largely depends on aerobic glycolysis. This metabolic switch optimizes glucose consumption in HSCs and redirects them to support fibrogenic transdifferentiation. ${ }^{2}$ As activated HSCs dynamically regulate the pathophysiology of chronic liver disease, modulation of cell fate and metabolism of HSCs has potential therapeutic implications for liver fibrosis.
Many signaling pathways control the growth and transdifferentiation of HSCs. These pathways become activated during various types of liver injury, and are presumed to modulate adult liver repair. ${ }^{3}$ Consistent with this notion, activation of hedgehog $(\mathrm{Hh})$ signaling, a highly conserved pathway orchestrating multiple aspects of development and tissue remodeling, has been documented in liver fibrosis. ${ }^{4} \mathrm{Hh}$ signaling is propagated by a family of ligands, namely Sonic hedgehog (Shh), Indian hedgehog, and Desert hedgehog, that bind to the membrane receptor Patched expressed on Hhresponsive cells. This interaction derepresses the activity of Patched on Smoothened (Smo), and permits the nuclear translocation of Glioblastoma (Gli) family transcription factors (Gli1, Gli2, and Gli3) that regulate the expression of Hh target genes. ${ }^{5}$ However, engagement of Hh ligands to

'Department of Pharmacology, School of Pharmacy, Nanjing University of Chinese Medicine, Nanjing, China; ${ }^{2}$ Jiangsu Key Laboratory for Pharmacology and Safety Evaluation of Chinese Materia Medica, Nanjing University of Chinese Medicine, Nanjing, China and ${ }^{3}$ The National First-Class Key Discipline for Traditional Chinese Medicine of Nanjing University of Chinese Medicine, Nanjing, China

Correspondence: Dr S Zheng, PhD, MD, Department of Pharmacology, School of Pharmacy, Nanjing University of Chinese Medicine, 138 Xianlin Avenue, Nanjing 210023 , Jiangsu, China.

E-mail: nytws@163.com

Received 23 December 2014; revised 13 February 2015; accepted 23 February 2015 
Patched can be prevented by hedgehog-interacting protein (Hhip), an endogenous antagonist of Hh ligands. ${ }^{5}$ Several studies demonstrate that $\mathrm{Hh}$ pathway activation promotes HSC activation and hepatic fibrogenesis, for example, accumulation of activated HSCs and extracellular matrix increases in parallel with progressive upregulation of $\mathrm{Hh}$ signaling in hepatotoxic diets-induced or cholestatic liver injury. ${ }^{6,7}$ Clinical evidence reveals that the severity of liver fibrosis parallels the level of Hh pathway activity in patients with different types of liver disease, including chronic viral hepatitis and nonalcoholic fatty liver disease. ${ }^{8}$

Natural products are an abundant source of antifibrogenic agents. Mounting studies demonstrate that curcumin is a promising therapeutic remedy for liver fibrosis. Curcumin protects hepatocytes against cellular damages induced by radiation ${ }^{9}$ and oxidative stress, ${ }^{10-12}$ and induces apoptosis of damaged hepatocytes. ${ }^{13}$ We demonstrate that curcumin reduces liver fibrosis by attenuating oxidative stress, suppressing inflammation, ${ }^{14}$ and inhibiting pathological angiogenesis. ${ }^{15}$ Curcumin also inhibits HSC activation associated with interruption of transforming growth factor- $\beta^{16}$ and plateletderived growth factor pathways. ${ }^{17}$ However, we hold that the underlying mechanisms are not sufficiently elucidated. Interestingly, latest investigations show that curcumin blocks Hh signaling leading to reversal of epithelial-mesenchymal transition in pancreatic cancer cells ${ }^{18}$ and induction of apoptosis in medulloblastoma cells. ${ }^{19}$ In this study, we hypothesize that modulation of Hh signaling could contribute to curcumin's antifibrotic activities including regulating cell fate, fibrogenic properties, and metabolism of HSCs. We therefore performed in vivo and in vitro experiments to test the hypothesis.

\section{MATERIALS AND METHODS}

\section{Reagents and Antibodies}

The following compounds were used in this study: curcumin and mitomycin (Sigma, St Louis, MO, USA), cyclopamine and SAG (Cayman, Ann Arbor, MI, USA), and H18 (Beyotime Biotechnology, Haimen, China). They were dissolved in dimethylsulfoxide (DMSO) for experiments. The following primary antibodies were used in this study: Patched, Glil, Cyclin D1, CDK4, Cyclin E1, CDK2, Bax, Pro-caspase-9, Cleaved-caspase-9, Pro-caspase-3, Cleaved-caspase-3, Pro-caspase-8, Cleaved-caspase-8, Full-length PARP-1, CleavedPARP-1, PPAR $\gamma$, and $\beta$-Actin (Cell Signaling Technology, Danvers, MA, USA); Smo, Hhip, Bcl-2 and CTGF (Santa Cruz Technology, Santa Cruz, CA, USA); $\alpha$-SMA, $\alpha 1$ (I)Procollagen, and Fibronectin (Epitomics, San Francisco, CA, USA); p-PKA, PKA, Lamin A/C, C/EBP $\alpha$, HK, PFK2, Glut 4, and MCT4 (Bioworld Technology, St Louis Park, MN, USA).

\section{Experimental Animal Procedures}

All experimental procedures were approved by the institutional and local committee on the care and use of animals of Nanjing University of Chinese Medicine (Nanjing, China), and all animals received humane care according to the
National Institutes of Health (USA) guidelines. Male SpragueDawley rats (200-250 g body weight) were obtained from Shanghai Slac Laboratory Animal (Shanghai, China). A mixture of carbon tetrachloride $\left(\mathrm{CCl}_{4} ; 0.1 \mathrm{ml}\right.$ per $100 \mathrm{~g}$ body weight) and olive oil $(1: 1(\mathrm{v} / \mathrm{v}))$ was used to induce liver fibrosis in rats. A total of 36 rats were randomly divided into six groups $(n=6)$. Group 1 was the vehicle control in which rats were not administrated $\mathrm{CCl}_{4}$ or curcumin but intraperitoneally (i.p.) injected with olive oil. Group 2 was the $\mathrm{CCl}_{4}$ group in which rats were i.p. injected with $\mathrm{CCl}_{4}$ without curcumin treatment. Group 3 was the positive control in which rats were injected with $\mathrm{CCl}_{4}$ and treated with colchicine (Yifeng Pharmacy, Nanjing, China) at $0.1 \mathrm{mg} / \mathrm{kg}$. Groups 4, 5, and 6 were treatment groups in which rats were i.p. injected with $\mathrm{CCl}_{4}$ and orally given curcumin at 100, 200, and $300 \mathrm{mg} / \mathrm{kg}$, respectively. Rats in groups 2-6 were i.p. injected with $\mathrm{CCl}_{4}$ every other day for 8 weeks. Curcumin and colchicine were suspended in sterile phosphate-buffered saline (PBS) and given once daily by gavage during weeks $5-8$. The control animals in groups 1 and 2 were similarly handled, including i.p. injection with the same volume of olive oil and oral administration of the same volume of PBS. At the end of experiments, rats were killed after being anesthetized by i.p. injection with pentobarbital $(50 \mathrm{mg} / \mathrm{kg})$. A small portion of the liver was removed for histopathological and immunohistochemical studies.

\section{Cell Culture}

Primary HSCs were isolated from male Sprague-Dawley rats as we previously described in detail. ${ }^{20}$ HSCs were cultured in Dulbecco's modified eagle medium (DMEM; Invitrogen, Grand Island, NY, USA) with 10\% fetal bovine serum (FBS; Sijiqing Biological Engineering Materials, Hangzhou, China) and $1 \%$ antibiotics, and grown in a $5 \% \mathrm{CO}_{2}$ humidified atmosphere at $37^{\circ} \mathrm{C}$. HSCs at passages 2-4 were used in experiments.

\section{Immunofluorescence Staining}

Immunofluorescence staining with liver tissues or treated cells were performed as we previously reported. ${ }^{15}$ 4',6-Diamidino2-phenylindole (DAPI) was used to stain the nucleus in liver tissues. Hoechst reagent was used to stain the nucleus of HSCs in vitro.

\section{Enzyme-Linked Immunosorbent Assay (ELISA)}

The levels of Shh in liver tissues and HSC culture supernatant were determined using an ELISA kit (Nanjing Jiancheng Bioengineering Institute, Nanjing, China) according to the protocol. Six duplicate wells were set up for each group. Results were from triplicate experiments.

\section{Cell Proliferation Assay}

Cell proliferation was evaluated using colorimetric BrdU cell proliferation enzyme-linked immunoassay kit (Nanjing Jiancheng Bioengineering Institute). HSCs were treated with 
various reagents at indicated concentrations with BrdU for $24 \mathrm{~h}$. BrdU incorporation in the proliferating cells was detected following manufacturer's instructions. Six duplicate wells were set up for each group. Results were from triplicate experiments.

\section{Analyses of Cell Cycle by Flow Cytometry}

HSCs treated with various reagents at indicated concentrations for $24 \mathrm{~h}$ were harvested and fixed. Cell cycle was detected using cellular DNA flow cytometric kits (Nanjing KeyGen Biotech, Nanjing, China) according to the protocol. Percentages of cells within cell cycle compartments (G0/G1, S, and G2/M) were determined by flow cytometry (FACSCalibur; Becton, Dickinson and Company, Franklin Lakes, NJ, USA). The data were analyzed using the software CellQuest. Results were from triplicate experiments.

\section{Analyses of Apoptosis}

HSCs were treated with various reagents at indicated concentrations for $24 \mathrm{~h}$. Morphology of apoptotic HSCs was evaluated using Hoechst staining kits and TUNEL staining kits (Nanjing Keygen Biotechnology) according to the protocols. Photographs were taken under a fluorescence microscope (Nikon, Tokyo, Japan). In addition, apoptotic rates were determined by flow cytometry using Annexin V-FITC apoptosis assay kits (Nanjing KeyGen Biotech) according to the protocol. Apoptotic cells were defined as the cells situated in the right two quadrants of each plot and the percentages were determined by flow cytometry (FACSCalibur; Becton, Dickinson and Company). The data were analyzed using the software CELLQuest. All these experiments were performed in triplicate.

\section{Oil Red $O$ Staining}

HSCs were treated with various reagents at indicated concentrations for $24 \mathrm{~h}$, and then were stained with oil red $\mathrm{O}$ to visualize the lipid droplets using the kits (Nanjing Jiancheng Bioengineering Institute) according to the protocol. Photographs were taken under a light microscope. Results were from triplicate experiments.

\section{Boyden Chamber Assay}

Polycarbonate membrane transwell inserts $(8 \mu \mathrm{m}$ pore size; Corning, USA) were coated with growth factor reduced Matrigel (BD Biosciences, Bedford, MA, USA) diluted with $\operatorname{DMEM}(1: 5, \mathrm{v} / \mathrm{v})$. HSCs were seeded to the upper wells and treated with various reagents at indicated concentrations. The lower chambers were filled with complete medium. After $24 \mathrm{~h}$ of incubation, the polycarbonated filter was removed and the migrated cells on the lower surface were stained with crystal violet. The number of migrated cells at five random fields for each well was counted and normalized to control. Results were from triplicate experiments.

\section{Wound Healing Assay}

Migration of HSCs was assessed by wounding healing assays and visualized using a real-time cell imaging system (IncuCyte live-cell ESSEN BioScience, Ann Arbor, MI, USA). HSCs were seeded in 96-well plates and grown to confluence, and then pretreated with mitomycin $(4 \mu \mathrm{g} / \mathrm{ml})$ for $3 \mathrm{~h}$. HSCs were resuspended in DMEM supplemented with 10\% FBS, and a scratch was made using a 96-pin Wound Maker. HSCs were treated with various reagents at indicated concentrations for $24 \mathrm{~h}$. During the experiment, typical kinetic updates were recorded at $2 \mathrm{~h}$ intervals, and the wound photographs were automatically obtained and registered by the IncuCyte software system. The data were expressed as percentage of wound confluence. Results were from triplicate experiments.

\section{Assessment of Mitochondria}

Mitochondria were visualized by staining with the fluorescent mitochondrion-selective probe MitoTracker (Invitrogen, Paisley, UK) in HSCs. Briefly, HSCs were seeded in 6-well plates, in which glass coverslips were placed onto each well, and cultured in DMEM supplemented with 10\% FBS for $24 \mathrm{~h}$. Cells were treated with various reagents at indicated concentrations for $24 \mathrm{~h}$. Cells were then washed with PBS and fixed with $4 \%$ paraformaldehyde for $40 \mathrm{~min}$ followed by incubation with the red probe added to DMEM for $40 \mathrm{~min}$. Subsequently, the nucleus was stained with Hoechst reagent. Photographs were taken under a fluorescence microscope. In addition, flow cytometry was used to quantify the mitochondrial fluorescence intensity. Briefly, treated HSCs were harvested and washed with PBS, and then fixed with $4 \%$ paraformaldehyde for $40 \mathrm{~min}$. Cells were resuspended in PBS at a density of $1 \times 10^{6} / \mathrm{ml}$ and incubated with the red probe for $40 \mathrm{~min}$. Cells were then subjected to flow cytometry analyses. Results were from triplicate experiments.

\section{Measurement of Energy Metabolic Parameters}

Intracellular levels of adenosine triphosphate (ATP) (Beyotime Institute of Biotechnology, Haimen, China) and lactate (Nanjing KeyGen Biotech), and activities of HK (Nanjing Jiancheng Bioengineering Institute) and PFK2 (Nanjing Jiancheng Bioengineering Institute) in lysates of treated HSCs were determined using their corresponding kits according to the manufacturers' instructions, respectively. Results were from triplicate experiments.

\section{Measurement of Glut 4 Membrane Translocation by Flow Cytometry}

Membrane translocation of Glut 4 was determined by flow cytometry according to reported methods. ${ }^{21}$ Briefly, HSCs were treated with various reagents at indicated concentrations for $24 \mathrm{~h}$. Cells were trypsinized and suspended in DMEM, and then incubated with the primary antibody against Glut 4 (1:100 dilution) at $4{ }^{\circ} \mathrm{C}$ for $24 \mathrm{~h}$ followed by incubation with FITC-labeled Goat Anti-Rabbit IgG (1:50 dilution; Wuhan Boster Biological Technology, Wuhan, China) for $2 \mathrm{~h}$ at room 
temperature. The intensity of cellular fluorescence was determined by flow cytometry (FACSCalibur; BD). Results were from triplicate experiments.

\section{Electrophoretic Mobility Shift Assay (EMSA)}

Nuclear extracts from treated HSCs were prepared using the NE-PER Nuclear Protein Extraction Kit (Pierce, Rockford, IL, USA) according to the protocol. Biotin-labeled Gli1 probe was prepared using an EMSA kit in accordance with the manufacturer's instructions (Pierce). The extracted nuclear protein $(10 \mathrm{mg})$ were incubated in a binding reaction mixture containing $1.5 \mathrm{ml} 10 \times$ binding buffer, $1.5 \mathrm{ml}$ poly $(\mathrm{dI}-\mathrm{dC})$ $(1.0 \mathrm{mg} / \mathrm{ml})$, and $\mathrm{ddH}_{2} \mathrm{O}$ to a final volume of $14.4 \mathrm{ml}$ for $20 \mathrm{~min}$ at room temperature. Then, the probe of $0.6 \mathrm{ml}$ ( $300 \mathrm{fmol}$ ) was added and incubated for $20 \mathrm{~min}$ at room temperature. Where indicated, $2 \mathrm{ml}$ of specific, cold-competitor oligonucleotides in $100 \times$ competing buffer was added before the labeled probe, and the reaction was incubated for $20 \mathrm{~min}$. Protein-DNA complexes were subject to electrophoresis in a $6.5 \%$ acrylamide gel at $4{ }^{\circ} \mathrm{C}$ for $1 \mathrm{~h}$. The gels were transferred to the bonding membrane at room temperature for $40 \mathrm{~min}$. After crosslinking for $10 \mathrm{~min}$ with an ultraviolet crosslinking apparatus, the membrane was blocked, streptavidin-HRP labeled, washed again, and equilibrated. Images were captured using the Gel Doc2000 system (Bio-Rad, USA).

\section{Real-time PCR}

Total RNA was prepared from treated HSCs using Trizol reagent (Sigma) following the protocol provided by the manufacturer. Real-time PCR was performed as we described previously. ${ }^{22}$ Glyceraldehyde phosphate dehydrogenase $(\mathrm{GAPDH})$ was used as the invariant control. Fold changes in the mRNA levels of target genes related to the invariant control GAPDH were calculated according to the suggested method. ${ }^{23}$ The following primers of genes (GenScript, Nanjing, China) were used: cyclin D1: (forward) 5'-TGGAGC CCCTGAAGAG-3', (reverse) 5'-AAGTGCGTTGTGCGGTA GC-3'; cyclin D2: (forward) 5'-GAGAAGCTGTCCCTGAT CCGCAAGC-3'， (reverse) 5'-AGACTTGGAGCCGTTGTG CTGCTC-3'; Bcl-2: (forward) 5'-CTGGTGGCAACATCGCT CTG-3', (reverse) 5'-GGTCTGCTGACCTCACTTGTG-3'; GAPDH: (forward) 5'-GGCCCCTCTGGAAAGCTGTG-3', (reverse) 5'-CCGCCTGCTTCACCACCTTCT-3'. Results were from triplicate experiments.

\section{Western Blot Analyses}

Whole cell protein extracts were prepared from treated HSCs with radioimmunoprecipitation assay buffer to which phenylmethylsulfonyl fluoride and/or phosphatase inhibitor was added immediately after addition of the lysis buffer. In certain experiments, nuclear proteins and cytoplasmic proteins were separated using a Bioepitope Nuclear and Cytoplasmic Extraction Kit (Bioworld Technology, St Louis Park, MN, USA) according to the protocol. Protein detection and band visualization and densitometry were performed as we previously described..$^{15} \beta$-Actin was used as an invariant control for equal loading of total proteins, and Lamin A/C was for nuclear proteins. Representative blots were from three independent experiments.

\section{Statistical Analysis}

Data were presented as mean \pm s.d. and analyzed using GraphPad Prism 5 software (San Diego, CA, USA). The significance of difference was determined by one-way ANOVA with the post hoc Dunnett's test. Values of $P<0.05$ were considered to be statistically significant.

\section{RESULTS}

\section{Curcumin Inhibits Hh Signaling in Rat Fibrotic Liver}

Our prior data have sufficiently demonstrated that curcumin protected the liver from histological injury, pathological angiogenesis and fibrogenesis induced by chronic $\mathrm{CCl}_{4}$ injection in rats. ${ }^{14,15,20}$ In this study, we initially examined the key molecules of Hh signaling in rat fibrotic liver. Results from immunofluorescence staining showed that Patched, Smo, and Hhip were expressed in HSCs indicated by double staining, respectively, with $\alpha$-smooth muscle actin ( $\alpha$-SMA), the marker of HSC activation. Specifically, the expression of Patched and Smo was elevated in rat fibrotic liver, but was reduced by treatment with colchicine and curcumin at various doses; however, the expression of Hhip was decreased in the fibrotic liver, but was restored by colchicine and curcumin (Figure 1). Moreover, chronic $\mathrm{CCl}_{4}$ intoxication increased the production of Shh in rat fibrotic liver, but curcumin at 200 and $300 \mathrm{mg} / \mathrm{kg}$ significantly reduced the levels of Shh in liver tissues (Supplementary Figure 1). Taken together, these data indicated that curcumin inhibited $\mathrm{Hh}$ signaling in $\mathrm{CCl}_{4}$ induced fibrotic liver in vivo that was associated with reduction of HSC activation and alleviation of liver fibrosis.

\section{Curcumin Blocks Hh Signaling in HSCs}

We next investigated the effects of curcumin on Hh signaling in cultured HSCs. Measurement of Shh levels in supernatant of HSCs showed that curcumin dose-dependently reduced Shh secretion and at $30 \mu \mathrm{M}$ produced a significant inhibitory effect compared with the control (Figure 2a). Western blot analyses demonstrated that curcumin downregulated Patched and Smo but upregulated Hhip at the protein level (Figure 2b). It is known that $\mathrm{Hh}$ signal transduction primarily depends on the transcriptional activity of Gli1, whose nuclear translocation, in turn, is negatively regulated by phosphorylation of protein kinase A (PKA). ${ }^{5}$ We found that PKA phosphorylation was increased by curcumin in HSCs (Figure 2c), suggesting that Glil nuclear translocation could be halted. Expectedly, immunofluorescence double staining showed that Gli1 accumulated in cytoplasm upon curcumin treatment (Figure 2d). Determination of Gli1 distribution by western blot analyses revealed that curcumin dose-dependently increased Gli abundance in cytoplasm and decreased its 

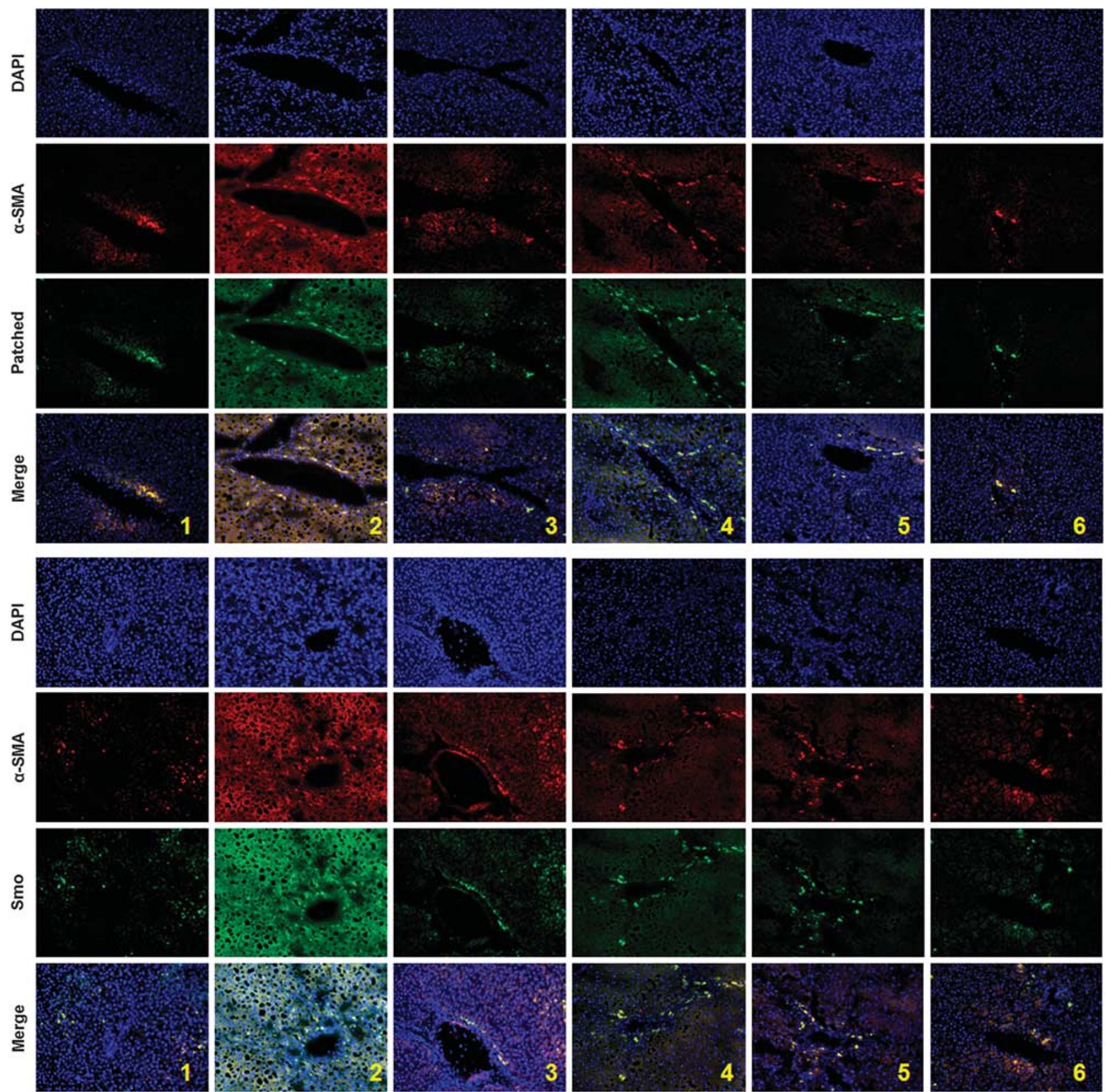

Figure 1 Curcumin inhibits $\mathrm{Hh}$ signaling in rat fibrotic liver. Rats were grouped as follows: group 1, vehicle control (no $\mathrm{CCl}_{4}$, no treatment); group 2, model group (with $\mathrm{CCl}_{4}$, no treatment); group 3, colchicine-treated group $\left(0.1 \mathrm{mg} / \mathrm{kg}+\mathrm{CCl}_{4}\right)$; group 4, curcumin-treated group (100 mg/kg+CCl $)$; group 5, curcumin-treated group $\left(200 \mathrm{mg} / \mathrm{kg}+\mathrm{CCl}_{4}\right)$; and group 6, curcumin-treated group $\left(300 \mathrm{mg} / \mathrm{kg}+\mathrm{CCl}_{4}\right)$. Liver sections were stained with immunofluorescence using antibodies against Patched, Smo, and Hhip. Antibody against $a$-SMA was used to specifically stain HSCs, and DAPI to stain the nucleus.

abundance in nucleus (Figure 2e). The interaction of Gli1 with DNA sequence was also significantly reduced by curcumin as demonstrated by the EMSA data (Figure 2f). Furthermore, curcumin dose-dependently downregulated the mRNA levels of cyclin D1, cyclin D2, and Bcl-2, three wellestablished target genes of Hh signaling in mammal cells ${ }^{24}$ (Figure 2g). In addition, PKA inhibitor H89 diminished curcumin prevention of Glil translocation (Supplementary Figure 2). Hh signaling agonist SAG rescued curcumin inhibition of Hh pathway (Supplementary Figure 3). Taken together, these findings consistently revealed that curcumin blocked Hh signaling in HSCs in vitro.

\section{Disruption of Hh Signaling Is Required for Curcumin Inhibition of Cell Growth and Induction of Apoptosis in HSCs}

We subsequently explored whether blockade of Hh signaling contributed to curcumin reduction of HSC activation. 

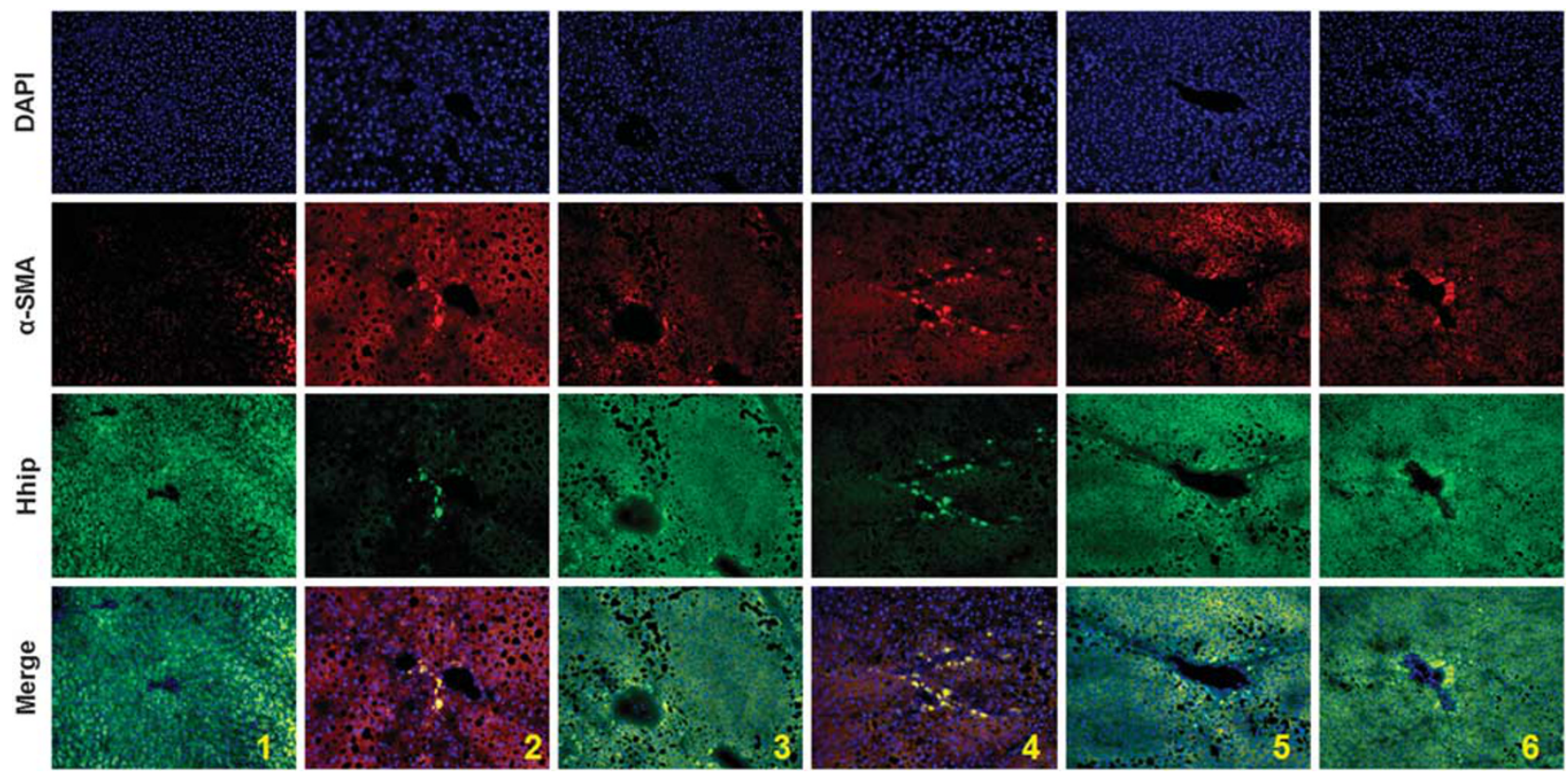

Figure 1 Continued.

We previously reported that curcumin inhibited cell proliferation and arrested cell cycle in HSCs. ${ }^{25}$ Herein, we demonstrated that $\mathrm{Hh}$ signaling specific inhibitor cyclopamine at $10 \mu \mathrm{M}$ and curcumin at $20 \mu \mathrm{M}$ significantly suppressed HSC proliferation, but SAG rescued curcumin inhibition of HSC proliferation (Figure 3a). Cyclopamine also significantly induced G0/G1 cell cycle arrest mimicking the curcumin effects in HSCs, but SAG weakened the curcumin effects (Figure $3 \mathrm{~b}$ ). Cell cycle is regulated by multiple cyclins and cyclin-dependent kinases (CDKs). Cyclin D1/CDK4 complex and cyclin E1/CDK2 complex jointly promote the G0- to S-phase transition. ${ }^{26}$ We observed that cyclopamine and curcumin downregulated the four molecules, but SAG abolished the curcumin effects on these molecules (Figure 3c). These data indicated that curcumin arrested G0/G1 cell cycle arrest by inhibiting Hh signaling in HSCs. Furthermore, induction of apoptosis was another important aspect for curcumin reduction of HSC activation. ${ }^{27}$ We assessed the morphology of apoptosis using TUNEL staining (Figure 3d) and Hoechst staining (Supplementary Figure 4) and observed that cyclopamine, similar to curcumin, induced HSC apoptosis, but SAG weakened the pro-apoptotic effects of curcumin. Consistent results were provided by quantitative evaluation of apoptotic rate by flow cytometry (Figure 3e). Further examinations of apoptosis-regulatory proteins showed that cyclopamine dose-dependently upregulated the pro-apoptotic Bax, downregulate the anti-apoptotic Bcl-2, and activated the caspase cascade and poly ADP-ribose polymerase-1 (PARP-1), mimicking the curcumin effects on these molecules (Figure 3f). These findings collectively suggested that disruption of $\mathrm{Hh}$ signaling was required for curcumin induction of HSC apoptosis.

\section{Disruption of Hh Signaling Is Required for Curcumin Inhibition of Fibrogenic Properties in HSCs}

Activated HSCs exhibit a series of fibrogenic behaviors including secretion of several pro-fibrogenic proteins, loss of lipid droplets, and enhanced capacity of invasion and migration in the pathogenesis of liver fibrosis. ${ }^{1} \mathrm{We}$ previously demonstrated that curcumin significantly downregulated fibrotic marker genes leading to inhibited HSC activation. ${ }^{28}$ In this study, cyclopamine was found to dose-dependently reduce the expression of $\alpha$-SMA, $\alpha 1$ (I) procollagen, fibronectin, and connective tissue growth factor (CTGF) and strengthen the inhibitory effects of curcumin on these genes (Figure 4a). Both cyclopamine and curcumin increased the expression of CCAAT/enhancer-binding protein $\alpha(\mathrm{C} / \mathrm{EBP} \alpha)$ and peroxisome proliferator-activated receptor $\gamma$ (PPAR $\gamma)$, two key molecules for maintaining the lipocyte phenotype and quiescence of $\mathrm{HSCs},{ }^{29}$ but SAG abolished the curcumin upregulation of these two molecules (Figure $4 \mathrm{~b}$ ). These results were confirmed by oil red $\mathrm{O}$ staining that visually examined the lipid content in HSCs (Figure 4c). Furthermore, we performed transwell invasion assay and wound healing assay to evaluate the motility of HSCs treated with curcumin and/or Hh signaling modulators. Results showed that cyclopamine and curcumin significantly suppressed the invasion and migration of HSCs, but curcumin effects were significantly rescued by SAG (Figure $4 \mathrm{~d}$ and e). Taken together, these discoveries indicated that interruption of $\mathrm{Hh}$ signaling was associated with inhibition of fibrogenic gene expression, restoration of intracellular lipid, and reduction of motility by curcumin in HSCs. 




d
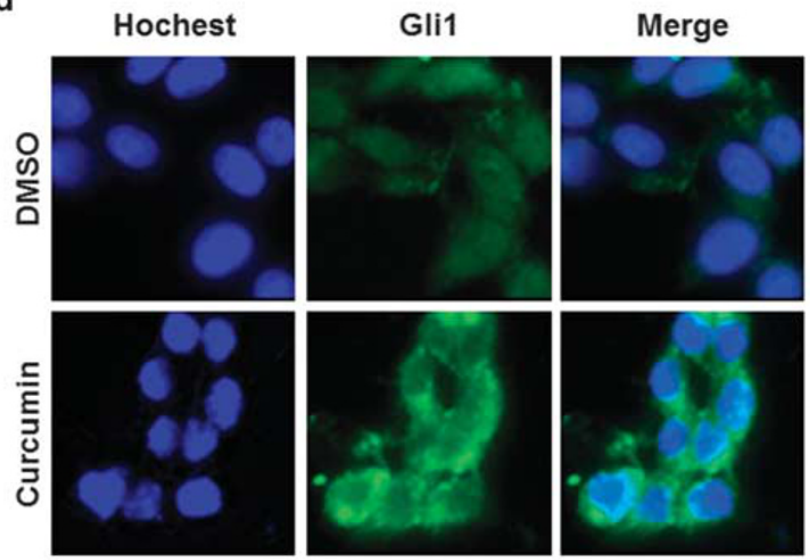

e

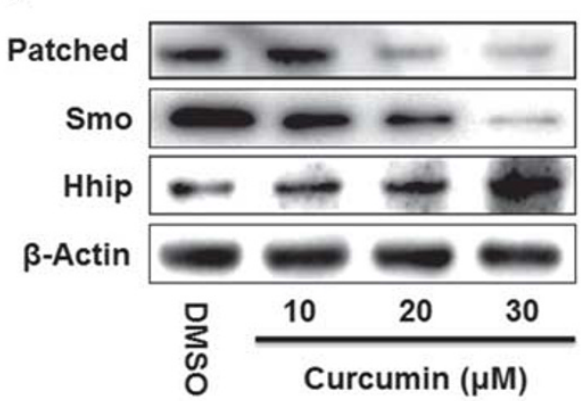

c

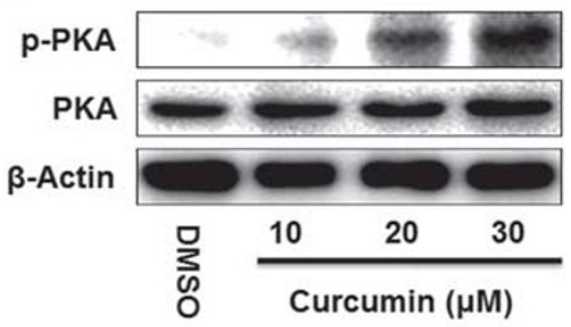

f
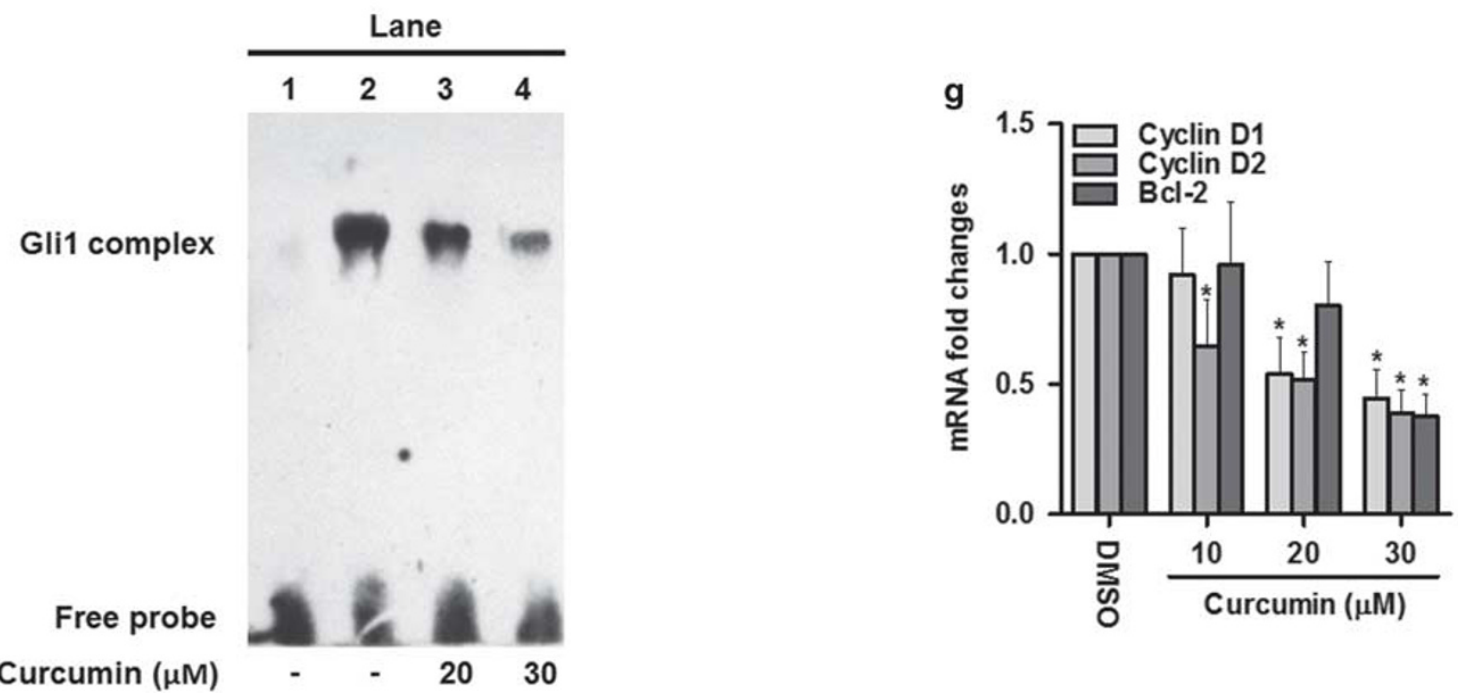

Figure 2 Curcumin blocks Hh signaling in HSCs. HSCs were treated with DMSO $(0.02 \%$, w/v) and curcumin at indicated concentrations for 24 h. (a) Shh levels in supernatant were examined by ELISA. Significance: ${ }^{*} P<0.05$ vs DMSO. (b) Western blot analyses of protein expression of Patched, Smo, and Hhip. (c) Western blot analyses of phosphorylation of PKA. (d) Immunofluorescence using antibody against Gli. Hoechst reagent was used to stain the nucleus. (e) Western blot analyses of protein abundance of Gli in the cytoplasm and nucleus, respectively. (f) EMSA for examining the binding capacity of Gli1 to DNA sequences. Lane 1 indicates samples treated with probe alone. Lane 2 was samples treated with DMSO without curcumin. (g) Real-time PCR analyses of Hh signaling target genes cyclin D1, cyclin D2, and Bcl-2. Significance: ${ }^{*} P<0.05$ vs DMSO.

\section{Curcumin Inhibits Glycolysis by Blocking Hh Signaling in HSCs}

Increasing studies demonstrate that glucose catabolism for energy supply is primarily dependent on anaerobic glycolysis during HSC activation. ${ }^{2}$ To test the curcumin effects on energy metabolism, we first examined the status of mitochondria, the undertaker for oxidative phosphorylation, using fluorescence staining and quantitative analyses. Interestingly, 
a

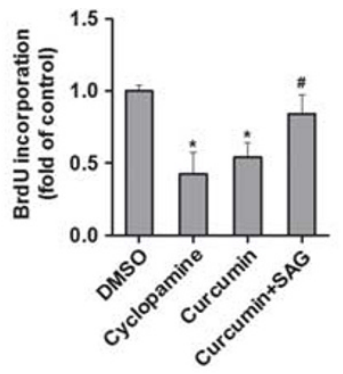

c

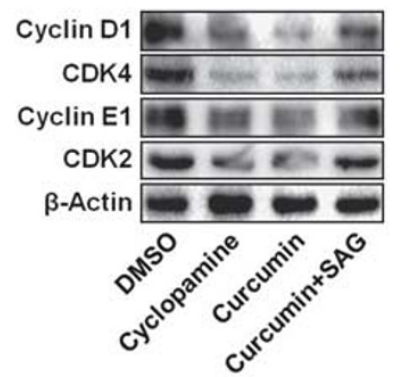

b

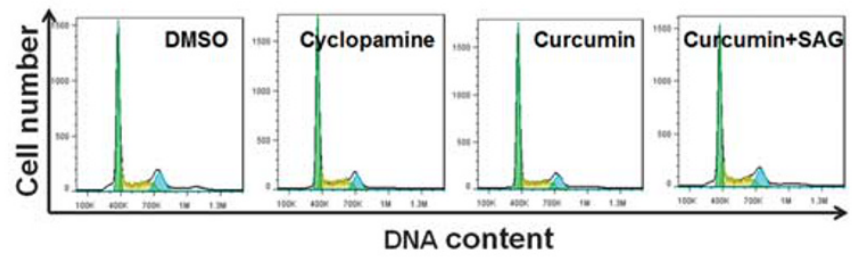

d

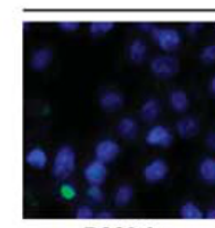

DMSO

TUNEL staining

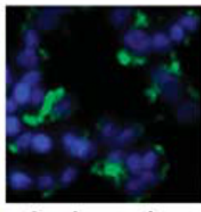

Cyclopamine

e

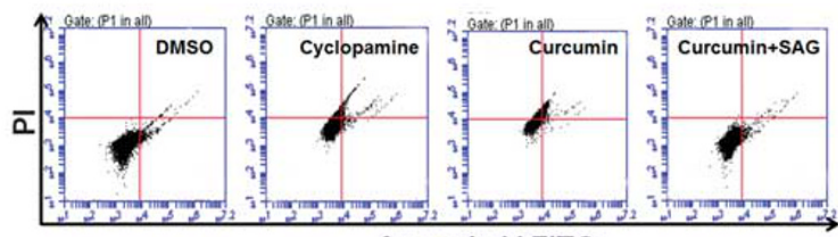

Annexin V-FITC

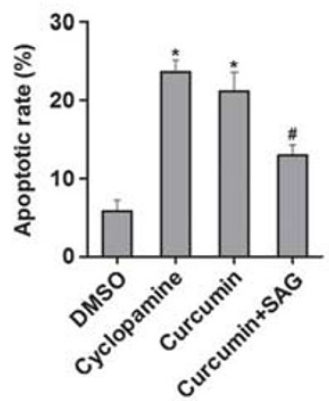

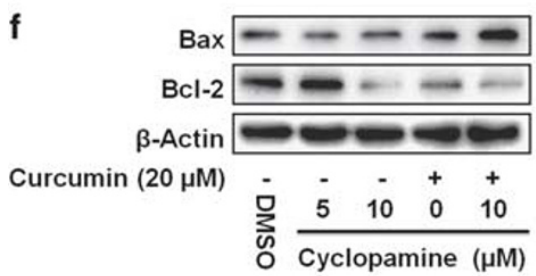

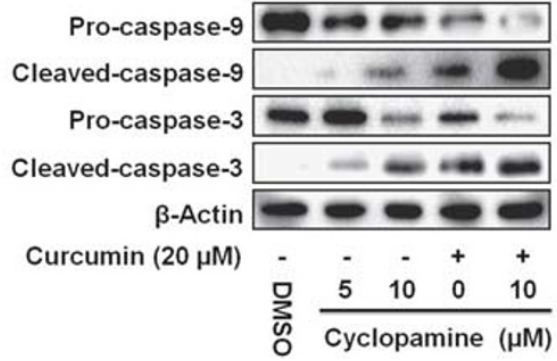

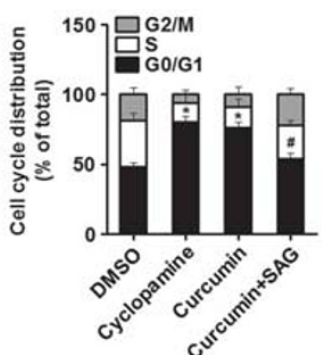

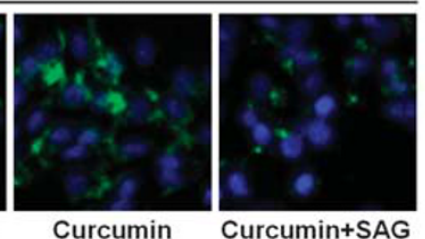

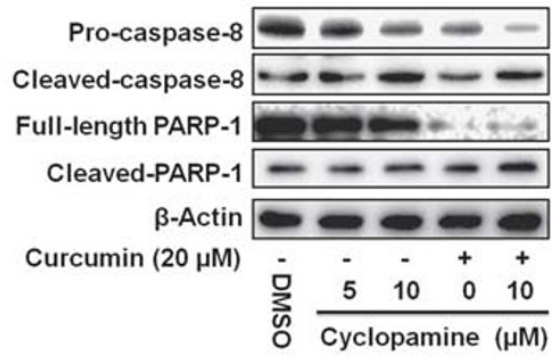

Figure 3 Disruption of Hh signaling is required for curcumin inhibition of cell growth and induction of apoptosis in HSCs. HSCs were treated with DMSO $(0.02 \%, w / v)$, cyclopamine $(10 \mu \mathrm{M})$, curcumin $(20 \mu \mathrm{M})$, and/or SAG $(0.6 \mu \mathrm{M})$ for $24 \mathrm{~h}$. (a) BrdU incorporation assay for evaluating cell proliferation. Significance: ${ }^{*} P<0.05$ vs DMSO, ${ }^{\#} P<0.05$ vs curcumin. (b) Cell cycle analysis by flow cytometry. Percentages of cell cycle distributions were determined. Significance: ${ }^{*} P<0.05$ vs DMSO, ${ }^{\#} P<0.05$ vs curcumin. (c) Western blot analyses of cell cycle-regulatory proteins cyclin D1, CDK4, cyclin E1, and CDK2. (d) TUNEL staining for evaluating apoptosis. Green fluorescence indicates apoptotic cells. (e) Flow cytometry analyses of apoptosis using FITC-labeled Annexin-V/PI staining. Cells situated in the right two quadrants of each plot were regarded as apoptotic cells. Significance: ${ }^{*} P<0.05$ vs DMSO, ${ }^{\#} P<0.05$ vs curcumin. (f) Western blot analyses of apoptosis-regulatory proteins including Bcl-2 family proteins, caspase cascade, and PARP-1.

curcumin and cyclopamine did not affect the number of mitochondria in HSCs (Figure $5 \mathrm{a}$ and $\mathrm{b}$ ), indicating that $\mathrm{Hh}$ signaling might not control oxidative phosphorylation in HSCs. In turn, we investigated the effects of curcumin on glycolysis and the role of Hh signaling in curcumin effects. We detected the intracellular levels of ATP and lactate, two products of glycolysis, and the activities of hexokinase (HK) and phosphofructokinase-2 (PFK2), two rate-limiting enzymes in glycolysis, ${ }^{2}$ demonstrating that curcumin decreased these parameters dose-dependently and cyclopamine produced similar inhibitory effects (Figure 5c). Western blot analyses consistently showed that curcumin and cyclopamine inhibited 
a

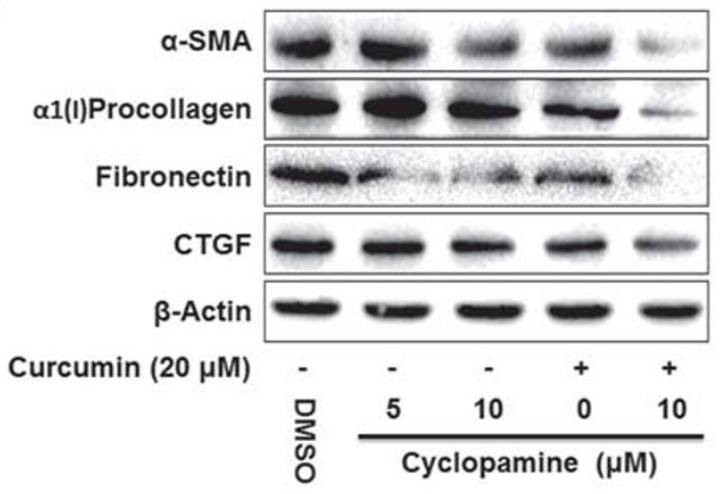

b

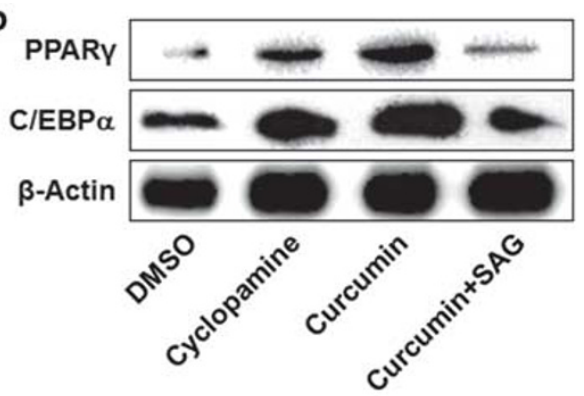

C Oil red O staining

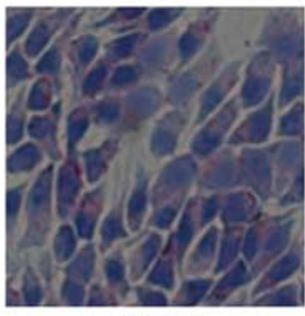

DMSO

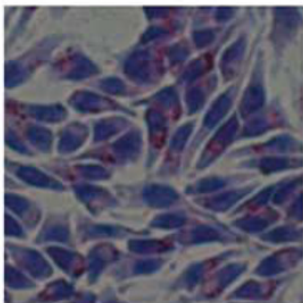

Cyclopamine

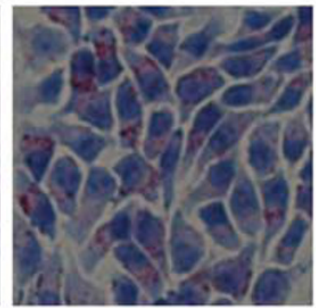

Curcumin

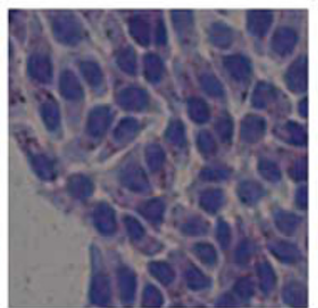

Curcumin+SAG d

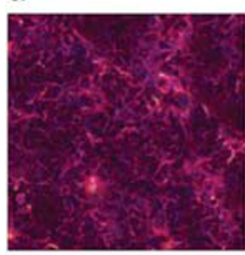

DMSO

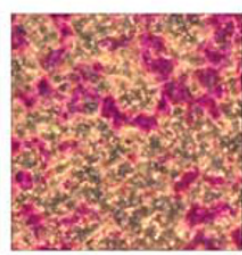

Cyclopamine



Curcumin

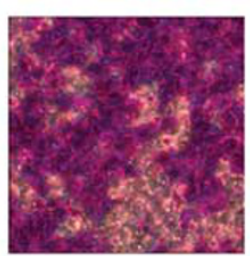

Curcumin+SAG

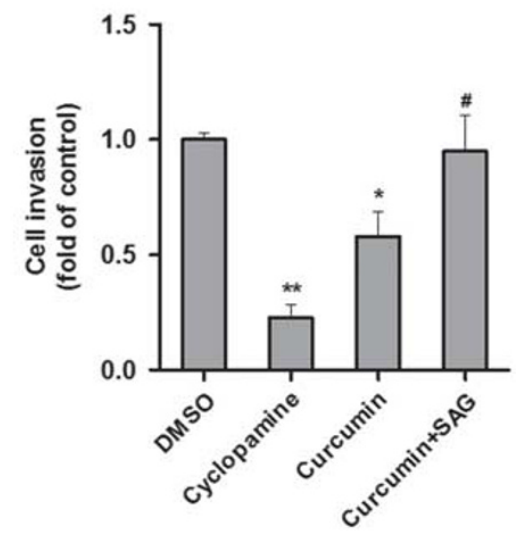

e

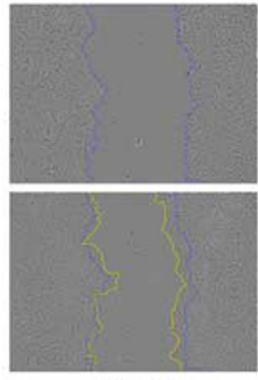

DMSO


Cyclopamine
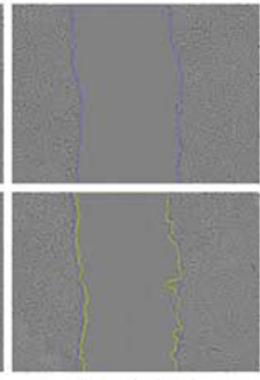

Curcumin

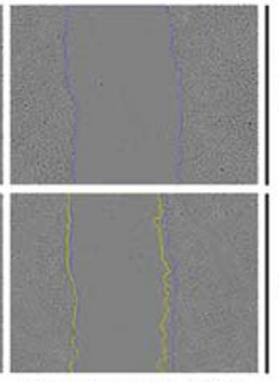

Curcumin+SAG

Figure 4 Disruption of Hh signaling is required for curcumin inhibition of fibrogenic properties in HSCs. HSCs were treated with DMSO (0.02\%, w/v), cyclopamine $(5$ or $10 \mu \mathrm{M})$, curcumin $(20 \mu \mathrm{M})$, and/or SAG $(0.6 \mu \mathrm{M})$ for $24 \mathrm{~h}$. (a) Western blot analyses of protein expression of fibrogenic molecules $a$-SMA, a1(I)procollagen, fibronectin, and CTGF. (b) Western blot analyses of protein expression of PPARY and C/EBPa. (c) Oil red O staining for evaluating lipid accumulation. (d) Transwell invasion assay with quantification. Significance: ${ }^{*} P<0.05$ vs DMSO, ${ }^{* *} P<0.05$ vs DMSO, ${ }^{\#} P<0.05$ vs curcumin. (e) Wound healing assay for evaluating cell migration with quantification. Significance: ${ }^{*} P<0.05$ vs DMSO, ${ }^{\#} P<0.05$ vs curcumin. 




DMSO

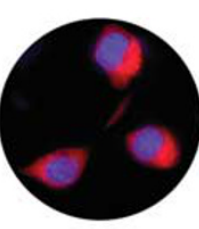

10

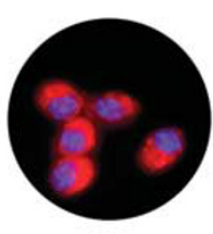

20

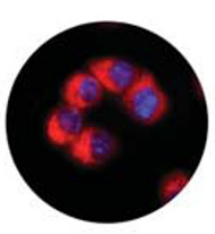

10 b



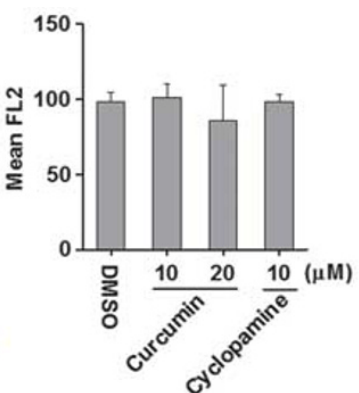

C
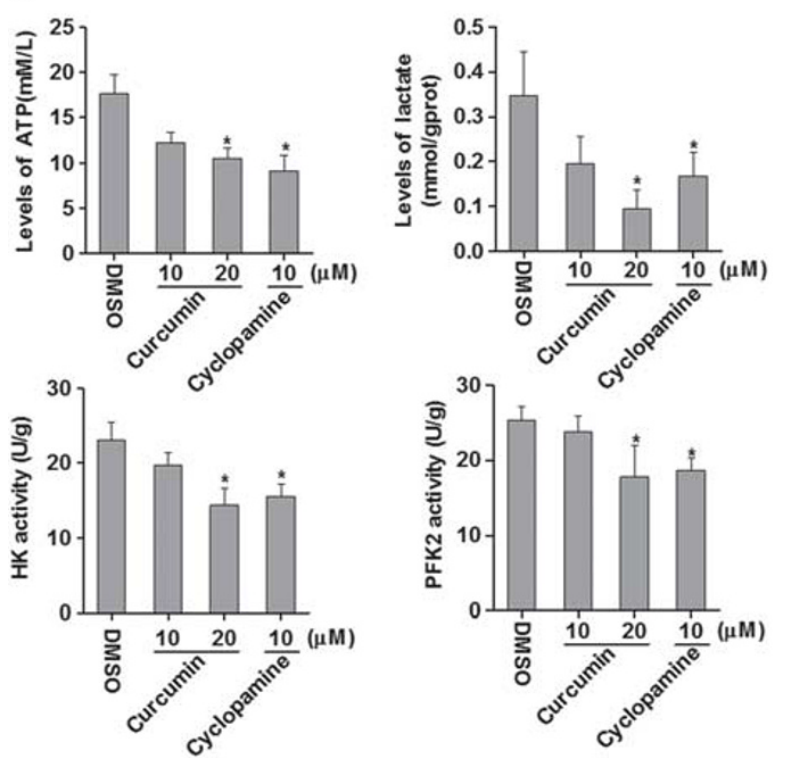

e

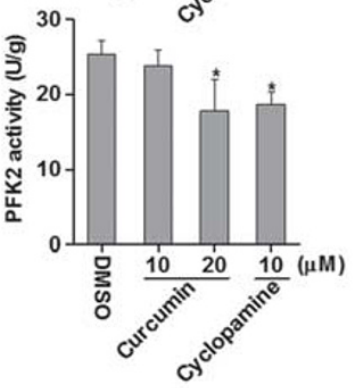

Curcumin $(20 \mu \mathrm{M})$ !

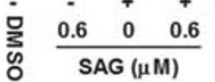

Curcumin $(20.0$
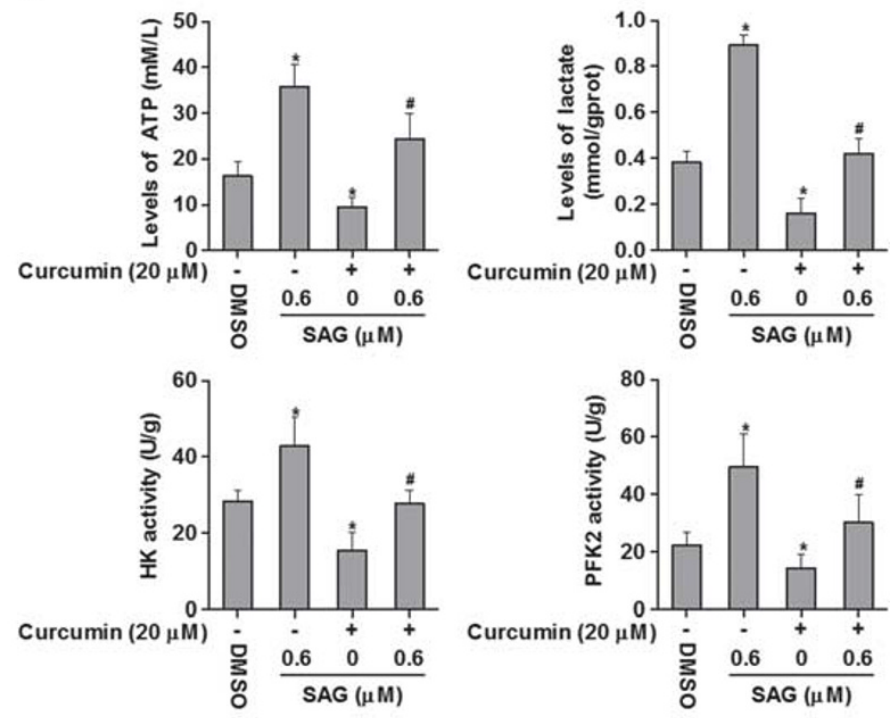

d

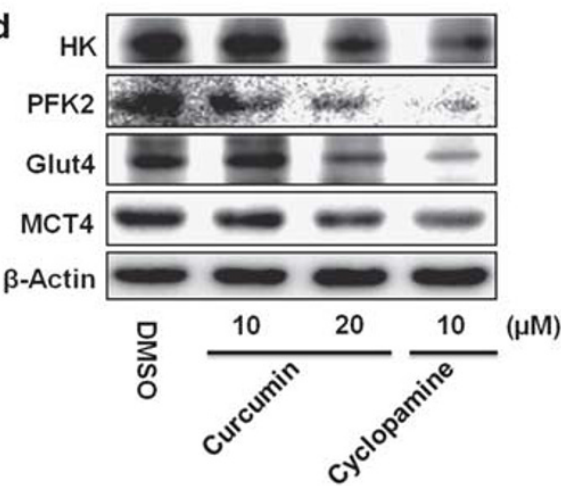

g
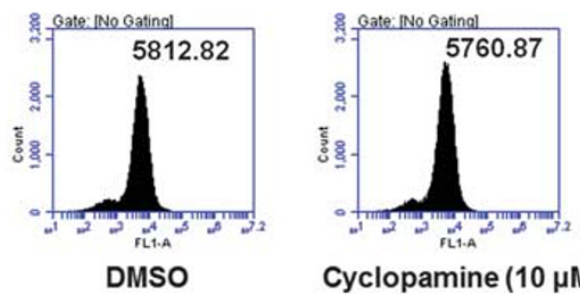

Cyclopamine $(10 \mu \mathrm{M})$



Curcumin $(20 \mu \mathrm{M})$
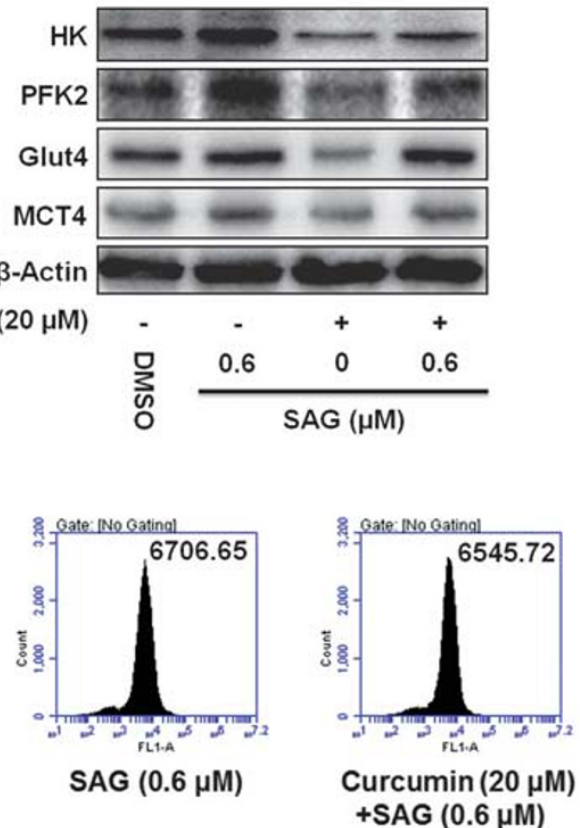

Figure 5 See for caption on page 800 . 
Curcumin inhibits Hh signaling in HSCs

N Lian et al

Figure 5 Curcumin inhibits glycolysis by blocking Hh signaling in HSCs. HSCs were treated with DMSO (0.02\%, w/v), cyclopamine, curcumin, and/or SAG at indicated concentrations for $24 \mathrm{~h}$. (a) Assessment of mitochondria using fluorescent mitochondrion-selective probe indicated by red fluorescence. Hoechst reagent was used to stain the nucleus. (b) Quantification of mitochondrial fluorescence intensity using flow cytometry. (c) Measurement of energy metabolic parameters including intracellular levels of ATP and lactate, and activities of HK and PFK2 by ELISA. Significance: *P<0.05 vs DMSO. (d) Western blot analyses of key molecules HK, PFK2, Glut4, and MCT4 in glycolysis. (e) Measurement of energy metabolic parameters including intracellular levels of ATP and lactate, and activities of HK and PFK2 by ELISA. Significance: ${ }^{*} P<0.05$ vs DMSO, " $P<0.05$ vs curcumin. (f) Western blot analyses of key molecules HK, PFK2, Glut4, and MCT4 in glycolysis. (g) Flow cytometry analyses of Glut4 membrane translocation. The mean fluorescence intensity is indicated in the top right corner of each plot.

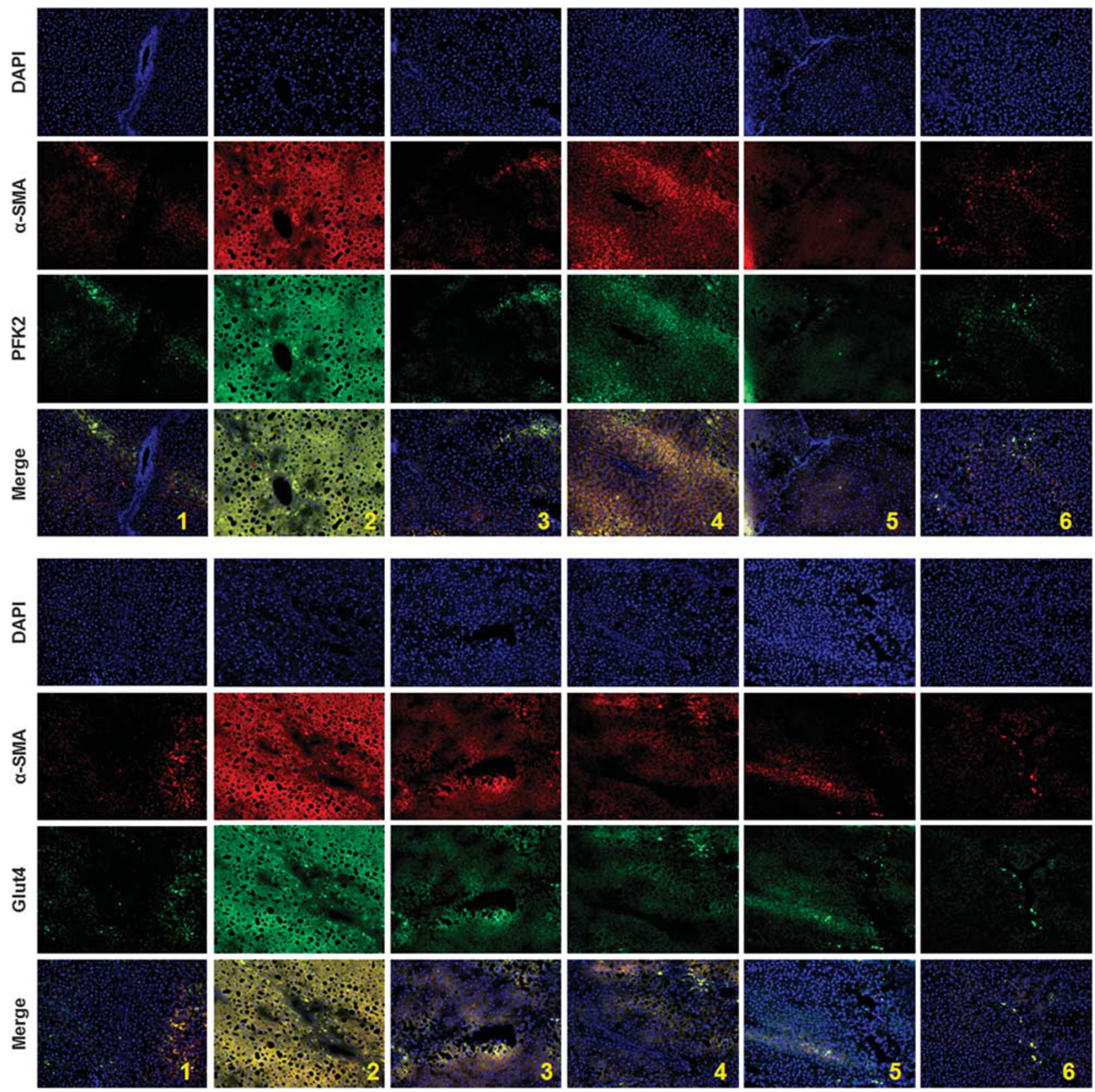

Figure 6 Curcumin reduces PFK2 and Glut4 expression in rat fibrotic liver. Rats were grouped as follows: group 1, vehicle control (no $\mathrm{CCl}_{4}$, no treatment); group 2, model group (with $\mathrm{CCl}_{4}$, no treatment); group 3, colchicine-treated group $\left(0.1 \mathrm{mg} / \mathrm{kg}+\mathrm{CCl}_{4}\right)$; group 4, curcumin-treated group $\left(100 \mathrm{mg} / \mathrm{kg}+\mathrm{CCl}_{4}\right) ;$ group 5, curcumin-treated group $\left(200 \mathrm{mg} / \mathrm{kg}+\mathrm{CCl}_{4}\right)$; and group 6, curcumin-treated group $\left(300 \mathrm{mg} / \mathrm{kg}+\mathrm{CCl}_{4}\right)$. Liver sections were stained with immunofluorescence using antibodies against PFK2 and Glut4. Antibody against $a$-SMA was used to specifically stain HSCs, and DAPI to stain nucleus. 
HK and PFK2 at the protein level (Figure 5d). Moreover, glucose transporter 4 (Glut4) and lactate transporter MCT4 (monocarboxylate transporter 4) were also concomitantly downreguated by curcumin and cyclopamine (Figure 5d). These data collectively indicated that curcumin suppressed glycolysis in HSCs. Finally, SAG was used to test whether these effects of curcumin were associated with blockade of $\mathrm{Hh}$ signaling. We found that curcumin inhibition of ATP, lactate, $\mathrm{HK}$, and PFK2 was rescued by SAG (Figure 5e), and that curcumin reduction of protein abundance of HK, PFK2, Glut4, and MCT4 was also weakened by SAG (Figure 5f). In addition, flow cytometry analyses showed that Glut4 membrane translocation was suppressed by cyclopamine and curcumin, but enhanced by SAG; however, curcumin effect was abolished by SAG (Figure 5g). Results from animal experiments provided in vivo evidence that PFK2 and Glut4 were highly expressed in activated HSCs during liver fibrosis, but their expression was decreased by treatment with curcumin (Figure 6), indicating that curcumin inhibited glycolysis in HSCs in vivo. Collectively, these data revealed that curcumin inhibited glycolysis by blocking Hh signaling in HSCs.

\section{DISCUSSION}

Emerging evidence reveals that Hh pathway critically regulates growth and repair responses in adult liver. Understanding of the mechanisms underlying $\mathrm{Hh}$ ligand production indicates that Hh signaling would likely be activated when major hepatic reconstruction is required in adulthood. Hepatic production of Hh ligands and accumulation of Hh-target cells generally increase with the severity of hepatic injury and fibrosis. $^{30,31}$ Accumulation of Hh-responsive cells is not merely an epiphenomenon concomitant with liver fibrogenesis. Rather, such cells participate actively in adult liver remodeling. Our present animal experiments demonstrated that several key molecules in Hh pathway colocalized with the marker of activated HSCs in rat fibrotic liver and that numbers of activated HSCs closely paralleled the level of Shh. This confirms the observation that HSCs are Hh-responsive target cells and their transdifferentiation is coupled with dramatic downregulation of Hhip expression. ${ }^{32}$ These findings also provide rationale for targeting $\mathrm{Hh}$ pathway for hepatic fibrosis treatment. Studies showed that Hh-neutralizing antibodies drastically reduced the viability of activated HSCs and virtually eliminated the proliferative effects of mitogens for HSC activation. ${ }^{33}$ In addition, pharmacological inhibition of Hh pathway reverted activated HSCs to be a less myofibroblastic phenotype. ${ }^{33}$ These discoveries strongly support the possibility of treating liver fibrosis by blocking $\mathrm{Hh}$ pathway. Herein, we showed that curcumin reduced the expression of Patched and Smo, but rescued Hhip expression in rat fibrotic liver and in cultured HSCs. Most of the biological effects of Hh pathway result from transcriptional activity of Gli1 that generally activates transcription of Hhtarget genes. ${ }^{34}$ In current experiments, nuclear translocation, binding to DNA sequence, and target gene transcription of Gli1 were all significantly halted in curcumin-treated HSCs. These data consistently revealed that curcumin disrupted $\mathrm{Hh}$ signaling in activated HSCs. Given the potent hepatoprotective and antifibrotic effects, we postulated that inhibition of Hh pathway could be required for curcumin to reduce HSC activation and liver fibrosis.

Suppression of HSC proliferation and induction of apoptosis in activated HSCs have been proposed as therapeutic strategies for management of hepatic fibrosis. Xu and coworkers previously reported that curcumin inhibited HSC proliferation associated with cell cycle arrest and downregulation of cyclin D1. ${ }^{25}$ The results in the present study demonstrated that these actions of curcumin were dependent on inhibition of Hh pathway, because cyclopamine mimicked the curcumin effects, but SAG abolished them. Similar results were obtained in examination of apoptotic machinery, an essential element of cell cycle checkpoints allowing for selective removal of damaged cells. Blockade of Hh signaling was found to be required for curcumin modulation of Bcl-2 family proteins and activation of caspase cascade leading to cellular fragmentation in HSCs. Our findings were consistent with the concept that Hh pathway is a key part of a complex signaling network that engages other fundamental cell-fate regulators to orchestrate global changes in Hh-target cells. ${ }^{24}$ Several studies showed that Hh signaling promoted the expression of cyclin D1 and cyclin E, serving as a principal regulator of cell cycle. ${ }^{35,36}$ There was also evidence that Gli1 transcriptionally regulated the $\mathrm{Bcl}-2$ promoter, identifying Bcl-2 as a direct target gene of Hh pathway. ${ }^{37}$ Moreover, a recent investigation showed that cell cycle arrest at G0/G1 checkpoint and Bcl-2-mediated apoptosis were induced by pharmacological blockade of $\mathrm{Hh}$ signaling by resveratrol in pancreatic cancer cells. ${ }^{38}$ Similarly, our current data indicated that curcumin inhibition of $\mathrm{Hh}$ pathway modulated cyclins/CDKs and apoptosis-regulatory molecules concurrently leading to cell cycle arrest and mitochondrial apoptosis in HSCs. These results strengthen the notion that inhibition of Hh pathway could be a strategy for cell-fate regulation in HSCs.

The myofibroblastic phenotype of activated HSCs is characterized by overexpression of many pro-fibrogenic molecules. In this study, cyclopamine similar to curcumin downregulated the marker proteins of HSC activation, suggesting that $\mathrm{Hh}$ signaling was involved in curcumin elimination of fibrotic gene expression in HSCs. The process of HSC activation is similar to that of adipocyte dedifferentiation, accompanied by loss of lipid storage capacity and suppression of expression of transcription factors, including PPAR $\gamma$ and $\mathrm{C} / \mathrm{EBP} \alpha$, two master regulators in lipogenesis. ${ }^{39}$ These two transcription factors synergistically stimulate lipogenesis by cross-talking and mutual regulation. PPAR $\gamma$ activates $\mathrm{C} / \mathrm{EBP} \alpha$ that, in turn, exerts a positive feedback on the elevation and maintenance of PPAR $\gamma$ expression. ${ }^{40}$ Curcumin was reported to induce the expression of genes relevant to lipid 
accumulation and elevate the level of intracellular lipids in HSCs. ${ }^{29}$ Herein, we found that cyclopamine stimulated the expression of PPAR $\gamma$ and $\mathrm{C} / \mathrm{EBP} \alpha$ and accumulated intracellular lipids in HSCs. Similar results were observed in curcumin-treated HSCs. However, SAG abolished the curcumin effects, indicating that blockade of $\mathrm{Hh}$ pathway was required for curcumin upregulation of lipogenesis. Although a recent study linked $\mathrm{Hh}$ signaling to E2F1-dependent regulation of lipogenic enzymes in progenitor cells and medulloblastoma, ${ }^{41}$ modulation of lipid synthesis by $\mathrm{Hh}$ pathway has not been studied systematically. We postulated that Hh pathway might be critically involved in controlling HSC lipocyte phenotype during liver fibrogenesis, and this needs to be explored. Furthermore, enhanced motility is another fibrogenic behavior of activated HSCs. Migration and recruitment of HSCs into liver scar are important steps in the process of sinusoidal remodeling. Our current data showed that curcumin suppression of HSC capacity to invade and migrate was associated with interruption of Hh pathway. Consistent with this, Hh regulation of cell motility and inhibition of cell migration by blocking Hh signaling have been documented under various pathophysiological circumstances. ${ }^{42-45}$ Overall, Hh signaling could be a therapeutic target for reduction of HSC activation and inhibition of profibrogenic behaviors of activated HSCs.

Growing evidence suggests that HSCs undergo metabolic reprogramming during activation, redirecting them to glycolysis as a major means of energy supply, similar to the Warburg effect described in tumor cells. Reciprocal changes in glycolytic and gluconeogenic enzymes occur in activated HSCs to meet the metabolic requirements of rapid cell proliferation. Recent studies demonstrated that this metabolic switch was regulated by Hh pathway via inducing glycolysis and suppressing gluconeogenesis and lipogenesis. ${ }^{2}$ These metabolic perturbations resulted in lactate accumulation and strengthened the global reprogramming of gene expression to activate the high proliferative and fibrogenic properties of HSCs. This metabolism-centric mechanism has therapeutic implications for hepatic fibrosis and opens a new perspective to identify antifibrotic agents targeting HSC activation. In this study, we observed that the abundance of mitochondria was not apparently affected by curcumin and cyclopamine in HSCs, indicating that the capacity for oxidative phosphorylation was retained and might not be controlled by Hh pathway. However, curcumin and cyclopamine significantly decreased the accumulation of ATP and lactate, and downregulated the activities and expression of $\mathrm{HK}$ and PFK2 within HSCs. The glucose transporter Glut4 and lactate export pump MCT4 were also affected concurrently. These data indicated that curcumin had inhibitory effects on multiple aspects of glycolysis and regulated the metabolic reprogramming in activated HSCs, and they were also consistent with the recognition that Hh signaling regulated metabolism in activated HSCs. Our further observation that SAG abrogated the curcumin inhibition of glycolysis convincingly demonstrated that disruption of Hh pathway was required for curcumin modulation of metabolism in HSCs. Interestingly, curcumin inhibition of aerobic glycolysis and regulation of cell fate have also recently been documented in human colorectal cancer cells. ${ }^{46}$ It has been proposed that metabolic reprogramming of HSCs is a conserved response to liver injury and controls the fate of HSCs. Therefore, we postulated that regulation of cell cycle and apoptosis in HSCs by curcumin could be linked to its inhibition of glycolysis by blocking Hh pathway, and this needs further explorations.

In summary, the aggregate data in this study demonstrated that disruption of Hh signaling was required for curcumin to arrest cell cycle, induce apoptosis, and inhibit firbrogenic properties in HSCs. We also newly discovered that curcumin inhibited glycolysis and regulated metabolism by suppressing Hh pathway in HSCs. These findings provided novel molecular basis for the development of curcumin as an antifibrotic agent for liver fibrosis.

Supplementary Information accompanies the paper on the Laboratory Investigation website (http://www.laboratoryinvestigation.org)

\section{ACKNOWLEDGMENTS}

The financial support was from the National Natural Science Foundation of China (81270514, 31401210), the Youth Natural Science Foundation of Jiangsu Province (BK20140955), the Natural Science Research General Program of Jiangsu Higher Education Institutions (14KJB310011), the Youth Natural Science Foundation of Nanjing University of Chinese Medicine (13XZR20), the Project Funded by the Priority Academic Program Development of Jiangsu Higher Education Institutions (ysxk-2010), and the 2013 Program for Excellent Scientific and Technological Innovation Team of Jiangsu Higher Education.

\section{DISCLOSURE/CONFLICT OF INTEREST}

The authors declare no conflict of interest.

1. Yin $\mathrm{C}$, Evason $\mathrm{KJ}$, Asahina $\mathrm{K}$ et al. Hepatic stellate cells in liver development, regeneration, and cancer. J Clin Invest 2013;123:1902-1910.

2. Chen Y, Choi SS, Michelotti GA et al. Hedgehog controls hepatic stellate cell fate by regulating metabolism. Gastroenterology 2012;143: 1319-1329.

3. Hernandez-Gea V, Friedman SL. Pathogenesis of liver fibrosis. Annu Rev Pathol 2011;6:425-456.

4. Omenetti A, Choi S, Michelotti G et al. Hedgehog signaling in the liver. J Hepatol 2011;54:366-373.

5. Choi SS, Omenetti A, Syn WK et al. The role of Hedgehog signaling in fibrogenic liver repair. Int J Biochem Cell Biol 2011;43:238-244.

6. Chan IS, Guy CD, Machado MV et al. Alcohol activates the hedgehog pathway and induces related procarcinogenic processes in the alcohol-preferring rat model of hepatocarcinogenesis. Alcohol Clin Exp Res 2014;38:787-800.

7. Jung Y, McCall SJ, Li YX et al. Bile ductules and stromal cells express hedgehog ligands and/or hedgehog target genes in primary biliary cirrhosis. Hepatology 2007;45:1091-1096.

8. Guy CD, Suzuki A, Zdanowicz M et al. Hedgehog pathway activation parallels histologic severity of injury and fibrosis in human nonalcoholic fatty liver disease. Hepatology 2012;55:1711-1721.

9. Srinivasan M, Sudheer AR, Pillai KR et al. Modulatory effects of curcumin on gamma-radiation-induced cellular damage in primary culture of isolated rat hepatocytes. Environ Toxicol Pharmacol 2007;24:98-105.

10. Barreto R, Kawakita S, Tsuchiya J et al. Metal-induced oxidative damage in cultured hepatocytes and hepatic lysosomal fraction: beneficial effect of a curcumin/absinthium compound. Chin J. Dig Dis 2005;6: 31-36. 
11. Bao W, Li K, Rong $\mathrm{S}$ et al. Curcumin alleviates ethanol-induced hepatocytes oxidative damage involving heme oxygenase-1 induction. J Ethnopharmacol 2010;128:549-553.

12. Zhao SG, Li Q, Liu ZX et al. Curcumin attenuates insulin resistance in hepatocytes by inducing Nrf2 nuclear translocation. Hepatogastroenterology 2011;58:2106-2111.

13. Wang ME, Chen YC, Chen IS et al. Curcumin protects against thioacetamide-induced hepatic fibrosis by attenuating the inflammatory response and inducing apoptosis of damaged hepatocytes. J Nutr Biochem 2012;23:1352-1366.

14. Fu $\mathrm{Y}$, Zheng $\mathrm{S}$, Lin $\mathrm{J}$ et al. Curcumin protects the rat liver from CCl4-caused injury and fibrogenesis by attenuating oxidative stress and suppressing inflammation. Mol Pharmacol 2008; 73:399-409.

15. Zhang $F$, Zhang $Z$, Chen $L$ et al. Curcumin attenuates angiogenesis in liver fibrosis and inhibits angiogenic properties of hepatic stellate cells. J Cell Mol Med 2014;18:1392-1406.

16. Zheng $S$, Chen A. Disruption of transforming growth factor-beta signaling by curcumin induces gene expression of peroxisome proliferator-activated receptor-gamma in rat hepatic stellate cells. Am J Physiol Gastrointest Liver Physiol 2007;292:G113-G123.

17. Zhou $Y$, Zheng $S$, Lin J et al. The interruption of the PDGF and EGF signaling pathways by curcumin stimulates gene expression of PPARgamma in rat activated hepatic stellate cell in vitro. Lab Invest 2007;87:488-498.

18. Sun $X D$, Liu $X E$, Huang DS. Curcumin reverses the epithelialmesenchymal transition of pancreatic cancer cells by inhibiting the Hedgehog signaling pathway. Oncol Rep 2013;29:2401-2407.

19. Elamin $\mathrm{MH}$, Shinwari Z, Hendrayani SF et al. Curcumin inhibits the Sonic Hedgehog signaling pathway and triggers apoptosis in medulloblastoma cells. Mol Carcinog 2010:49:302-314.

20. Zhang Z, Guo Y, Zhang S et al. Curcumin modulates cannabinoid receptors in liver fibrosis in vivo and inhibits extracellular matrix expression in hepatic stellate cells by suppressing cannabinoid receptor type-1 in vitro. Eur J Pharmacol 2013;721:133-140.

21. Koshy S, Alizadeh P, Timchenko LT et al. Quantitative measurement of GLUT4 translocation to the plasma membrane by flow cytometry. J Vis Exp 2010;45:2429.

22. Zhang F, Ni C, Kong D et al. Ligustrazine attenuates oxidative stressinduced activation of hepatic stellate cells by interrupting plateletderived growth factor-beta receptor-mediated ERK and p38 pathways. Toxicol Appl Pharmacol 2012;265:51-60.

23. Schmittgen TD, Zakrajsek BA, Mills AG et al. Quantitative reverse transcription-polymerase chain reaction to study mRNA decay: comparison of endpoint and real-time methods. Anal Biochem 2000;285: 194-204.

24. Katoh $Y$, Katoh M. Hedgehog target genes: mechanisms of carcinogenesis induced by aberrant hedgehog signaling activation. Curr Mol Med 2009;9:873-886.

25. $\mathrm{Xu} \mathrm{J,Fu} \mathrm{Y,} \mathrm{Chen} \mathrm{A.} \mathrm{Activation} \mathrm{of} \mathrm{peroxisome} \mathrm{proliferator-activated}$ receptor-gamma contributes to the inhibitory effects of curcumin on rat hepatic stellate cell growth. Am J Physiol Gastrointest Liver Physiol 2003;285:G20-G30.

26. Satyanarayana A, Kaldis P. Mammalian cell-cycle regulation: several Cdks, numerous cyclins and diverse compensatory mechanisms. Oncogene 2009;28:2925-2939.

27. Zheng S, Chen A. Activation of PPARgamma is required for curcumin to induce apoptosis and to inhibit the expression of extracellular matrix genes in hepatic stellate cells in vitro. Biochem J 2004;384: 149-157.
28. Zheng S, Chen A. Curcumin suppresses the expression of extracellular matrix genes in activated hepatic stellate cells by inhibiting gene expression of connective tissue growth factor. Am J Physiol Gastrointest Liver Physiol 2006;290:G883-G893.

29. Tang Y, Chen A. Curcumin protects hepatic stellate cells against leptininduced activation in vitro by accumulating intracellular lipids. Endocrinology 2010;151:4168-4177.

30. Fleig SV, Choi SS, Yang L et al. Hepatic accumulation of Hedgehogreactive progenitors increases with severity of fatty liver damage in mice. Lab Invest 2007;87:1227-1239.

31. Omenetti $A$, Popov $Y$, Jung $Y$ et al. The hedgehog pathway regulates remodelling responses to biliary obstruction in rats. Gut 2008;57: 1275-1282.

32. Choi SS, Omenetti A, Witek RP et al. Hedgehog pathway activation and epithelial-to-mesenchymal transitions during myofibroblastic transformation of rat hepatic cells in culture and cirrhosis. Am J Physiol Gastrointest Liver Physiol 2009;297:G1093-G1106.

33. Yang $\mathrm{L}$, Wang $\mathrm{Y}, \mathrm{Mao} \mathrm{H}$ et al. Sonic hedgehog is an autocrine viability factor for myofibroblastic hepatic stellate cells. J Hepatol 2008;48: 98-106.

34. Katoh $\mathrm{Y}$, Katoh $\mathrm{M}$. Integrative genomic analyses on GLI1: positive regulation of GLI1 by Hedgehog-GLI, TGFbeta-Smads, and RTK-PI3KAKT signals, and negative regulation of GLI1 by Notch-CSL-HES/HEY, and GPCR-Gs-PKA signals. Int J Oncol 2009;35:187-192.

35. Duman-Scheel $M$, Weng $L$, Xin $S$ et al. Hedgehog regulates cell growth and proliferation by inducing Cyclin D and Cyclin E. Nature 2002;417: 299-304.

36. Li F, Duman-Scheel M, Yang D et al. Sonic hedgehog signaling induces vascular smooth muscle cell proliferation via induction of the G1 cyclinretinoblastoma axis. Arterioscler Thromb Vasc Biol 2010;30:1787-1794.

37. Bigelow RL, Chari NS, Unden $A B$ et al. Transcriptional regulation of bcl-2 mediated by the sonic hedgehog signaling pathway through gli-1. J Biol Chem 2004;279:1197-1205.

38. Mo W, Xu X, Xu L et al. Resveratrol inhibits proliferation and induces apoptosis through the hedgehog signaling pathway in pancreatic cancer cell. Pancreatology 2011;11:601-609.

39. Tsukamoto $\mathrm{H}$, She $\mathrm{H}$, Hazra $\mathrm{S}$ et al. Anti-adipogenic regulation underlies hepatic stellate cell transdifferentiation. J Gastroenterol Hepatol 2006;21:S102-S105.

40. Rosen ED, Walkey CJ, Puigserver $\mathrm{P}$ et al. Transcriptional regulation of adipogenesis. Genes Dev 2000;14:1293-1307.

41. Bhatia B, Hsieh M, Kenney AM et al. Mitogenic Sonic hedgehog signaling drives E2F1-dependent lipogenesis in progenitor cells and medulloblastoma. Oncogene 2011;30:410-422.

42. Bao C, Namgung $H$, Lee $J$ et al. Daidzein suppresses tumor necrosis factor-alpha induced migration and invasion by inhibiting hedgehog/ Gli1 signaling in human breast cancer cells. J Agric Food Chem 2014;62:3759-3767.

43. Samarzija I, Beard P. Hedgehog pathway regulators influence cervical cancer cell proliferation, survival and migration. Biochem Biophys Res Commun 2012;425:64-69.

44. Deshpande G, Swanhart L, Chiang $P$ et al. Hedgehog signaling in germ cell migration. Cell 2001;106:759-769.

45. Balordi F, Fishell G. Hedgehog signaling in the subventricular zone is required for both the maintenance of stem cells and the migration of newborn neurons. J Neurosci 2007;27:5936-5947.

46. Wang K, Fan H, Chen Q et al. Curcumin inhibits aerobic glycolysis and induces mitochondrial-mediated apoptosis through hexokinase II in human colorectal cancer cells in vitro. Anticancer Drugs 2015;26: 15-24. 